\title{
The basis of morality
}

\author{
Psychologists, anthropologists and biologists are uncovering the bigger picture behind the development \\ of empathy and altruism
}

\section{Philip Hunter}

M any philosophers and anthropologists might argue that among the attributes that make humans unique, it is our ability to reason morally that sets us apart from all other animalsperhaps in addition to our capacity for spirituality. From Ancient Greece and the Roman Republic, to sixth century China and the European Enlightenment; philosophers throughout the ages have pondered why humans can feel empathy or behave altruistically-and, indeed, why they even contemplate it. Only later, in the twentieth century, did the biological sciences join the quest for understanding by seeking genetic, neurological and evolutionary explanations of self-awareness and morality.

The answer has so far been elusive, as biologists have neither been able to find the 'moral' gene-if such a thing exists-nor to identify a specific cluster of neurons or region of the brain that takes care of ethical decision making. Yet, some parts of the picture are emerging and they reveal a relationship between the complex emotional processes that enable empathy and altruism and the advanced cognitive abilities, such as mirror self-recognition (MSR), that emerged with the evolution of the more complex structural and functional components of the brain. In addition, these studies show that many more animal species than we had appreciated, including birds, display more or less primitive versions of these traits.

These findings have led to a proliferation of research into 'human-like' social behaviour in animals. "Empathy research is really taking off, not only on adult humans in neuroscience or on children, but also animals," commented Dutch primatologist Frans de Waal, now at Emory University in Atlanta, GA, USA, who analyses the behaviour of chimpanzees to gain insight into their emotional and cognitive abilities. "We have collected thousands of observations of so-called consolation behaviour in chimpanzees. As soon as one among them is distressed, for example losing a fight, falling from a tree, or encountering a snake, others will come over to provide reassurance. They embrace the distressed chimp or try to calm him or her with a kiss and grooming. This behaviour is typical of chimps and other apes, and is used in research on children as the main behavioural marker of 'sympathetic concern'," he explained.

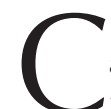

lues about the cognitive functions and neurological features underlying 'sympathetic concern' can be elucidated by correlating the results from studies of children with those of higher animals that appear capable of feeling empathy and possibly altruism. "In children, MSR emerges between 18-24 months of age and its onset is concurrent with the emergence of empathic behaviour and other indices of the theory of mind," commented Diana Reiss, a specialist in the evolution of intelligence at Hunter College of the City University of New York, USA. Reiss also pointed out that all species that exhibit MSR have large, complex brains relative to their body weight, with evidence of a developmental link between MSR and empathy, as in human children.

Researchers are now working to identify the neurological basis for this link, why it evolved and how it develops with the growing individual. MSR, widely regarded as an important requirement for empathic or altruistic behaviour, has now been identified in several species beyond the great apes and humans. Although MSR is not itself interesting from the perspective of cognitive evolution-it confers no direct selective advantage - as de Waal pointed out, the importance of the mirror test resides in what it tells us about the ability of animals to analyse their relationship with their environment, especially with respect to their social partners. "The mirror test is interesting not because it shows that an animal has the capacity for self-recognition but because of the cognitive abilities that are associated with MSR," he explained.

\section{...biologists have neither been able to find the 'moral' gene-if such a thing exists — nor to identify a specific cluster of neurons or region of the brain that takes care of ethical decision making}

Gordon Gallup originally demonstrated MSR in animals in 1970 (Gallup, 1970). He developed a test in which a visible coloured mark is made on a part of the animal's body that it cannot see without using a mirror. The test determines whether the animal can use the reflection to locate the mark and then touch or rub it, thus revealing that it can tell the reflection is of itself and not of another individual. Since Gallup's early experiments, MSR has been seen in a number of animals, most notably dolphins (Reiss \& Marino, 2001), the Asian elephant (Plotnik et al, 2006) and, a first for birds, the magpie (Prior et al, 2008).

$\Lambda$ Ithough MSR has now been demonstrated in several species, it is clearly confined to a small group with highly evolved social interactions. Most species in this group are also set apart from the rest 


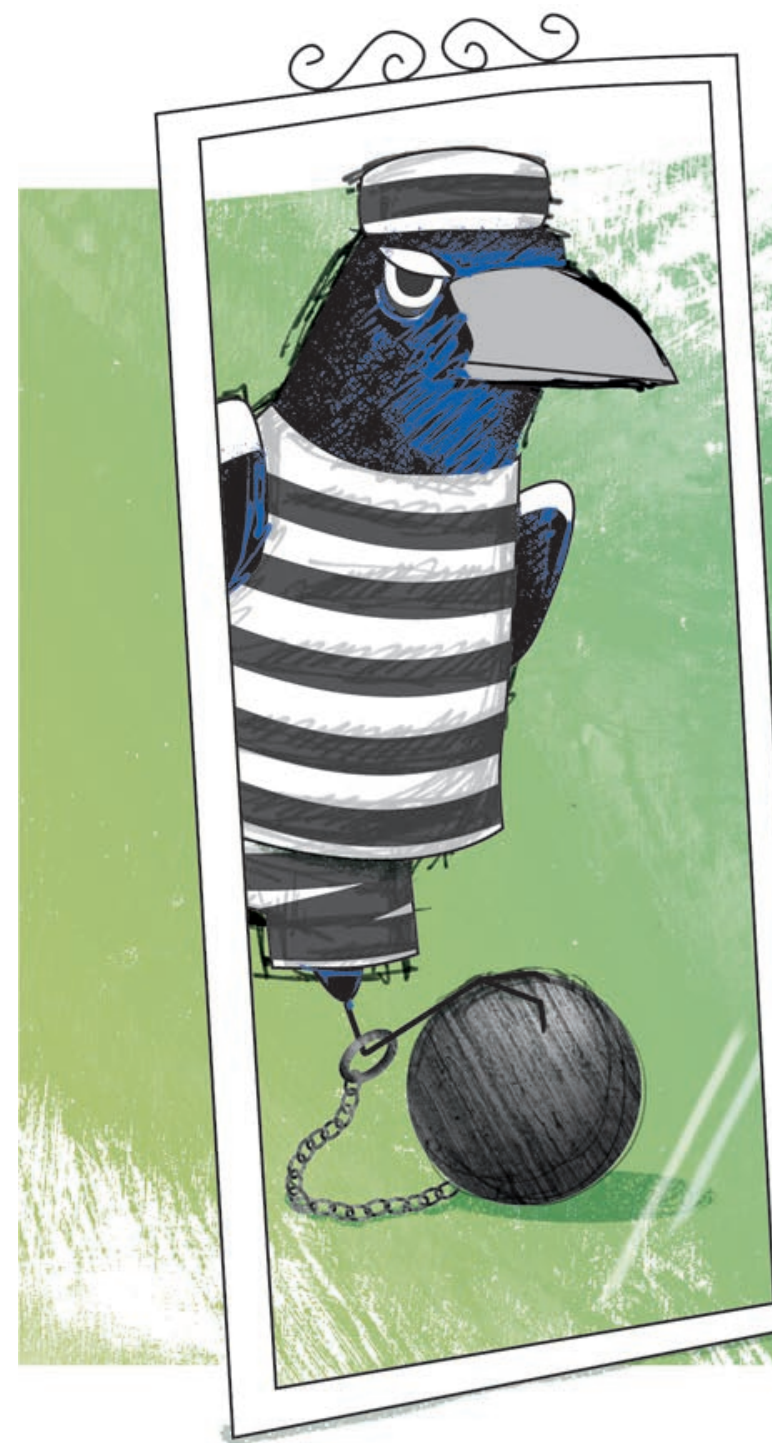

of the animal kingdom because they have spindle neurons, which are believed to be a vital component of large brains capable of empathic social behaviour. These neurons tend to be larger and have a streamlined bipolar structure that is well-adapted for the rapid and coordinated transmission of signals. Also known as von Economo neurons (VEN), they occur in areas of the cortex that process a large number of input signals from other brain regions, in particular the fronto-insular cortex and anterior cingulate cortex. Functionally, the VEN architecture seems to be optimized for the parallel receipt and processing of a large amount of diverse information.

"Recent research has reported that spindle neurons are found to occur only in the brains of a few species-humans, the great apes, whales, dolphins and elephants_leading to the speculation that they are a possible obligatory neuronal adaptation in very large

\section{Although MSR has now been demonstrated in several species, it is clearly confined to a small group with highly evolved social interactions}

brains, permitting fast information processing and transfer along highly specific projections and that evolved in relation to emerging social behaviours," commented Reiss, who was the first to demonstrate MSR in dolphins along with her colleague Lori Marino, also at Emory University. "It has been further suggested that these specialized brain cells may be involved in processing emotions and underlie empathic behaviour."

Furthermore, the absence of spindle neurons in all other primates suggests that these evolved independently among the great apes and other species that are capable of MSR. This finding leads to the question of whether the brains of higher social animals that have spindle neurons have further common structural or functional features that also evolved in parallel. A recent study suggests that this has happened, at least in the case of elephants and humans, both of which have similar extensive regions of neocortex, which is the neurological structure responsible for sensory perception, motor commands and higher level thought processes (Goodman et al, 2009). The study examined several mammalian species to record the number of nucleotide substitutions in genes that have a crucial role in the brain; the results showed that the most substitutions occur in humans and elephants. This suggests that elephant and human brains have both encountered strong selection for this particular group of 
genes, which the other animals included in the study clearly had not.

$\mathrm{H}$ owever, any suggestion that specific neurological structures are essential, at least for MSR, has been challenged by the discovery of MSR in magpies, which do not even have a neocortex. Although the detailed structures are different, the forebrains of magpies, along with some other members of the crow family, are large and have high neuron densities that are more comparable with humans than other bird species. Although spindle neurons have not yet been observed in magpies, their existence in birds has not been ruled out. In any case, it might be that magpies evolved more complex brain functions without the help of spindle neurons to speed up communication.

Nonetheless, there seems to be a clear correlation between the emergence of MSR and empathic behaviour. Magpies, for example, have been subject to extensive research precisely because their ecological conditions have driven the evolution of social intelligence (Prior et al, 2008). They steal and store food, but they also form stable partnerships based to some extent on trust, which requires discrimination between other individuals and judgments about their intentions.

\section{...the enhanced cognitive and social interactive abilities of dolphins and great apes have allowed these species to develop rudimentary levels of morality and altruism}

In the case of dolphins, the selective pressures they face are associated with their social groups - known as pods - which are able to cooperate with neighbouring groups in times of danger or when there is an opportunity to hunt for food on a larger scale. Dolphins are carnivores and have developed highly skilled cooperative techniques for hunting prey, similar to a pack of wolves hunting, but in three dimensions. Dolphins also devote a large amount of time to training their offspring to hunt and survive, and they are the only non-human mammals that exhibit strong evidence of vocal mimicry and physical imitation (Reiss et al, 1997).

Although they live in superficially different ecosystems, dolphins and the great apes have been subject to fundamentally similar selective pressures, according to Marino, who co-authored the seminal study of vocal mimicry in dolphins. "Despite the seemingly disparate environmental pressures that would shape cetacean-the mammalian group including dolphins, whales and porpoises-and primate cognitive evolution, these drivers were actually extremely similar," she said. "Both evolved complex levels of social interaction, and, in that respect, dolphins and primates evolved in, conceptually, very similar environments."

A ccording to Marino, dolphin brain enlargement occurred only when they began to develop the use of echolocation to coordinate hunting and other activities. But other animals have developed echolocation without enlarging their brains. In the case of cetaceans and especially dolphins, Marino believes the key evolutionary driver was the incorporation of this technique into a complex system of social interaction. She argues that the enhanced cognitive and social interactive abilities of dolphins and great apes have allowed these species to develop rudimentary levels of morality and altruism.

"Ongoing findings from studies of both primate and cetacean behaviour provide support for the conclusion that social complexity involves the evolution of morality," Marino explained. "This is true in a number of ways. From an ultimate evolutionarily point of view, complex group living requires ways to regulate interactions among individuals in order to keep the group intact. Frans de Waal has provided abundant evidence for this argument in primates and I would contend the same is true of cetaceans. On a proximate level, much of the morality we see in primate and cetacean groups is underwritten by empathy, which is enabled by the kind of awareness of self and other that dolphins and primates-particularly great apes and humans-demonstrate."

Such behaviour might have evolved partly because altruism enhanced individual survival chances-providing that the cost was not too great. But, according to Antonio Damasio, Director of the Brain and Creativity Institute at the University of Southern California in Los Angeles, CA, USA, the key driver might have been that altruism enabled individuals to make 'wiser' decisions. Humans might be unique in the degree to which we apply ethics

\section{...humans commonly appear to balance rational and emotional factors when making judgements, creating the impression that there are distinct neural systems competing with each other}

and morality to rational decision-making, but the presence of empathy in animals provides important clues about the neurological underpinnings of these abilities. In particular, research with animals might help to settle the long-standing argument about whether morality and altruism can be regarded as independent of the brain's hardware, or whether morality is hardwired into the brain and is a product of our neurological evolution.

$S^{\text {th }}$ ome studies seem to suggest that the latter is true. For example, an experiment at the University of Zurich, Switzerland, in which subjects were asked to divide up a sum of money on a supposedly fair basis, found that the disruption of cognition altered the moral behaviour of the participants. In the socalled Ultimatum Game, one of the two participants-who does not know how much money is available-is required to make an offer to the other, who does know the size of the pot. If the second player does not accept, neither player receives anything, so in a sense it can be seen as a test of indignation versus greed. If the pot is big, the second player might decide to swallow his or her pride and accept it. The first player, however, might decide to make a generous offer in order to be sure it would be accepted. During the game, the Swiss researchers applied low-frequency transcranial magnetic stimulation to disrupt either the right or left dorsolateral prefrontal cortex of their test subjects. Disruption of the right side made them more susceptible to 'immoral' decisions as they were more willing to make offers that they judged to be unfair to their partners. Yet, they retained their ability to determine fairness. The researchers concluded that the right side of the prefrontal cortex has an implicit role in determining how to apply moral or ethical standards to information made available by cognitive processes (Sanfey et al, 2003).

This suggests that morality is hardwired and does not exist independently of 
the brain's complex biochemistry, at least according to Damasio. "Emotions and feelings, as we know them, are related to our 'wet' biology, to our flesh," he said. "Formally you can construct them in robotic artefacts but there is no reason to believe [that] they would be the same."

I this regard, Michael Koenigs, from the Department of Psychiatry at the University of Wisconsin-Madison, USA, pointed out that humans commonly appear to balance rational and emotional factors when making judgements, creating the impression that there are distinct neural systems competing with each other. "From a psychological standpoint, in the midst of a sticky moral dilemma it can certainly feel like your mind is being pulled in two different directions," he said. "And in terms of the brain, it is very clear that certain areas are more concerned with emotion and affect, while other areas are more associated with 'cold' cognitive processes. Both systems play a role in determining morality, but at the neural level the relationship between the systems is more of an integration than a competition."

Until recently there was very little direct observation of these neurological processes at work. However, recent research based on functional magnetic resonance imaging has revealed that 'true' and 'false' statements activate different regions of the prefrontal cortex. The researchers found that people tend to use separate processes to resolve the distinctions between true and false statements, unless the answer is blindingly obvious (Marques et al, 2009). They concluded that people accept statements as true initially and confirm this merely by a call to memory. However, if the initial check suggests that the statement might be false, further reasoning is required to confirm the rejection. "The idea supported by this paper and others is that when the statement to verify is true-and by default we assume [that] it is-the task is to find if we have or recognize that information in our memory," explained Frederico Marques, one of the authors of the study from the University of Lisbon, Portugal. "When we do not find that information, or if we have doubts, we further process the information, more like problem solving."

Animals that are capable of MSR also possess primitive abilities to assess truth or falsehood. Magpies, for example, have to decide whether a particular individual can be trusted to not steal food. Of course, more research is needed to provide further insight into the neurological processes involved in such assessments and to illuminate the evolutionary history of such a skill. What is established, however, is that the roots of complex social behaviour and the capacity for abstract thought-as well as ethical judgment, perhaps — can be found predominantly in the more advanced warm-blooded and social vertebrates. The capacity for morality is perhaps not, after all, uniquely human.

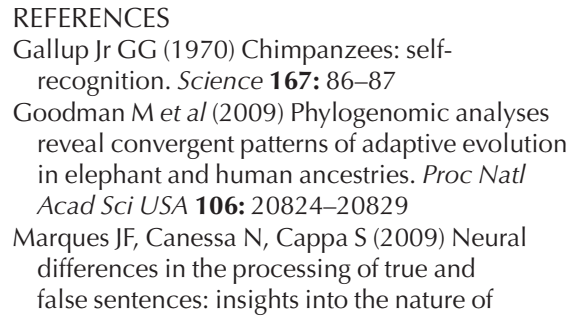

'truth' in language comprehension. Cortex 45: 759-768

Plotnik JM, de Waal FBM, Reiss D (2006) Selfrecognition in an Asian elephant. Proc Natl Acad Sci USA 103: 17053-17057

Prior H, Schwarz A, Güntürkün O (2008) Mirror-induced behavior in the magpie (Pica pica): evidence of self-recognition. PLOS Biol 6: e202

Reiss D, Marino L (2001) Mirror self-recognition in the bottlenose dolphin: a case of cognitive convergence. Proc Natl Acad Sci USA 98: 5937-5942

Reiss D, McCowan B, Marino L (1997)

Communicative and other cognitive characteristics of bottlenose dolphins. Trends Cogn Sci 1: 2

Sanfey AG, Rilling JK, Aronson JA, Nystrom LE, Cohen JD (2003) The neural basis of economic decision-making in the ultimatum game. Science 300: 1755-1758

\section{Philip Hunter is a freelance journalist} in London, UK.

EMBO reports (2010) 11, 166-169. doi:10.1038/embor.2010.19

\title{
Women and telomeres
}

\author{
Last year's Nobel Prizes for Carol Greider and Elizabeth Blackburn \\ should be encouraging for all female scientists with children
}

\section{Howard Wolinsky}

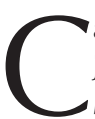

arol Greider, a molecular biologist at Johns Hopkins University (Baltimore, $M D$, USA), recalled that when she received a phone call from the Nobel Foundation early in October last year, she was staring down a large pile of laundry. The caller informed her that she had won the 2009 Nobel Prize in Physiology or Medicine along with Elizabeth Blackburn, her mentor and co-discoverer of the enzyme telomerase, and Jack Szostak. The Prize was not only the ultimate reward for her own achievements, but it also highlighted a research field in biology that, unlike most others, is renowned for attracting a significant number of women.

Indeed, the 2009 awards stood out in particular, as five women received Nobel prizes. In addition to the Prize for Greider and Blackburn, Ada E. Yonath received one in chemistry, Elinor Ostrom became the first female Prize-winner in economics, and Herta Müller won for literature (Fig 1).

Greider, the daughter of scientists, has overcome many obstacles during her career.

\section{The Prize [...] highlighted a research field in biology that, unlike most others, is renowned for attracting a significant number of women}

She had dyslexia that placed her in remedial classes; "I thought I was stupid," she told The New York Times (Dreifus, 2009). Yet, by far the biggest challenge she has tackled is being a woman with children in a man's world. When she attended a press conference at Johns Hopkins to announce the Prize, she brought her children Gwendolyn and Charles with her (Fig 2). "How many men have won the Nobel in the last few years, and they have kids the same age as mine, and their kids aren't in the picture? That's a big difference, right? And that makes a statement," she said.

Marie Curie (1867-1934), the PolishFrench physicist and chemist, was the first woman to win the Prize in 1903 for physics, 